

\title{
Electromagnetic calorimeter for the AMS-02 experiment
}

R. Kossakowski, F. Cadoux, V. Chambert-Hermel, G. Coignet, J.M. Dubois, D. Fougeron, N. Fouque, L. Girard, C. Goy, R. Hermel, et al.

\section{To cite this version:}

R. Kossakowski, F. Cadoux, V. Chambert-Hermel, G. Coignet, J.M. Dubois, et al.. Electromagnetic calorimeter for the AMS-02 experiment. International Conference on Calorimetry in Particle Physics 10 CALOR 2002, Mar 2002, Pasadena, United States. pp.108-113. in2p3-00021556

\section{HAL Id: in2p3-00021556 https://hal.in2p3.fr/in2p3-00021556}

Submitted on 2 Jun 2003

HAL is a multi-disciplinary open access archive for the deposit and dissemination of scientific research documents, whether they are published or not. The documents may come from teaching and research institutions in France or abroad, or from public or private research centers.
L'archive ouverte pluridisciplinaire HAL, est destinée au dépôt et à la diffusion de documents scientifiques de niveau recherche, publiés ou non, émanant des établissements d'enseignement et de recherche français ou étrangers, des laboratoires publics ou privés. 
LAPP-EXP 2002-14

December 2002

\section{Electromagnetic calorimeter for the AMS-02 experiment}

R. Kossakowski, F. Cadoux, V. Chambert-Hermel, G. Coignet, J.M. Dubois, D. Fougeron N. Fouque, L. Girard, C. Goy, R. Hermel, B. Lieunard, S. Rosier-Lees, J.P. Vialle

LAPP-IN2P3-CNRS, BP. 110, 74941 Annecy-le-Vieux Cedex, France

G. Chen, H. Chen, Z. Liu, Y. Lu, Z. Yu, H. Zhuang

IHEP, Chinese Academy of Science, 100039 Beijing, China

F. Cervelli, S. Di Falco, T. Lomtadze, G. Venanzoni,

INFN Sezione di Pisa, Via Livornese 1291, 56010 S. Piero a Grado, Italy

E. Falchini, P. Maestro, P.S. Marrocchesi, R. Paolietti, F. Pilo, N. Turini, G. Valle

INFN Siena Physics Dept., 55 v. Banchi di Sotto, 53100 Siena, Italy

Presented by R. Kossakowski

at CALOR 2002, Pasadena (USA), March 25-29, 2002 


\title{
ELECTROMAGNETIC CALORIMETER FOR THE AMS-02 EXPERIMENT
}

\author{
R. KOSSAKOWSKI, F. CADOUX, V. CHAMBERT-HERMEL, G. COIGNET, \\ J. M. DUBOIS, D. FOUGERON, N. FOUQUE, L. GIRARD, C. GOY, \\ R. HERMEL, B. LIEUNARD, S. ROSIER-LEES, J. P. VIALLE \\ $L A P P$ - BP 110, 74941 Annecy -le-Vieux Cedex, France \\ G. CHEN, H.CHEN, Z. LIU, Y. LU, Z. YU, H. ZHUANG \\ IHEP - Chinese Academy of Science, 100039 Beijing, China \\ F. CERVELLI, S. DI FALCO, T. LOMTADZE, G. VENANZONI \\ INFN - Sezione di Pisa, Via Livornese 1291, 56010 S. Piero a Grado, Italy \\ E. FAlChini, P. MAESTRO, P. S. MARROCCHESI, R. PAOLETTI, F. PILO, \\ N. TURINI, G. VALLE \\ Gruppo Collegato INFN - Siena Physics Dept.,55 v. Banchi di Sotto, 53100 Siena, Italy \\ (presented by R. Kossakowski at CALOR 2002, Email : kossakowski@lapp.in2p3.fr)
}

\begin{abstract}
The electromagnetic imaging calorimeter made of Lead and scintillating fibers will identify the high energy leptons and $\gamma$ rays in AMS-02 experiment on the International Space Station. Physics requirements and space qualification constraints lead to severe optimizations of the detector design, the mechanics and the electronics for this $16,5 X_{0}$ calorimeter sampled by 1296 electronic channels.
\end{abstract}

\section{Introduction}

The AMS- $02^{1}$ experiment aims at measuring the cosmic ray spectra in the range of energy from $\mathrm{GeV}$ to $\mathrm{TeV}$ for three years on the International Space Station ISS. The experimental set-up consists of a superconducting magnet, a silicon tracker and a number of additional detectors, designed to measure the energy and to identify the nature of cosmic rays. These detectors are: the transition radiation detector (TRD), the time of flight (TOF) which also provides a standard AMS trigger, the ring imaging Cerenkov counter (RICH) and the electromagnetic calorimeter (ECAL). The detailed description of the AMS-02 detector can be found elsewhere ${ }^{2}$. 
The electromagnetic calorimeter, which is being constructed by an Annecy (France), Beijing (China) and Pisa-Siena (Italy) collaboration, will be a major instrument to identify electrons, positrons and $\gamma$-rays and to measure their energy in particular in the high energy part of the spectrum. The ECAL is an imaging calorimeter consisting of 9 modules made of layers of Lead and scintillating fibers (Figure 1). Each module has a $648 \times 648 \mathrm{~mm}^{2}$ section and $18 \mathrm{~mm}$ depth, which corresponds to 1.8 radiation lengths. In two successive modules the fibers are rotated by 90 and follow on $\mathrm{X}$ or $\mathrm{Y}$ direction. The fibers of a module are read only at one end by the photomultiplier R7600-00-M4 from Hamamatsu ${ }^{3}$, placed alternatively on each side. One photomultiplier consists of 4 independent pixels. In this way the elementary cell of the calorimeter has the dimension of $648 \times 9 \mathrm{~mm}^{2}$ (or $9 \times 648 \mathrm{~mm}^{2}$ ) in X-Y directions and $9 \mathrm{~mm}$ in the $\mathrm{Z}$ direction. It corresponds roughly to a 0,5 Molire radius for the transverse dimension of the electromagnetic shower and to a 0,9 radiation length in the longitudinal direction. A particle impinging vertically on the ECAL crosses about 16,5 radiation lengths and the longitudinal profile of the electromagnetic shower is sampled by 18 independent measurements (Figure 1).

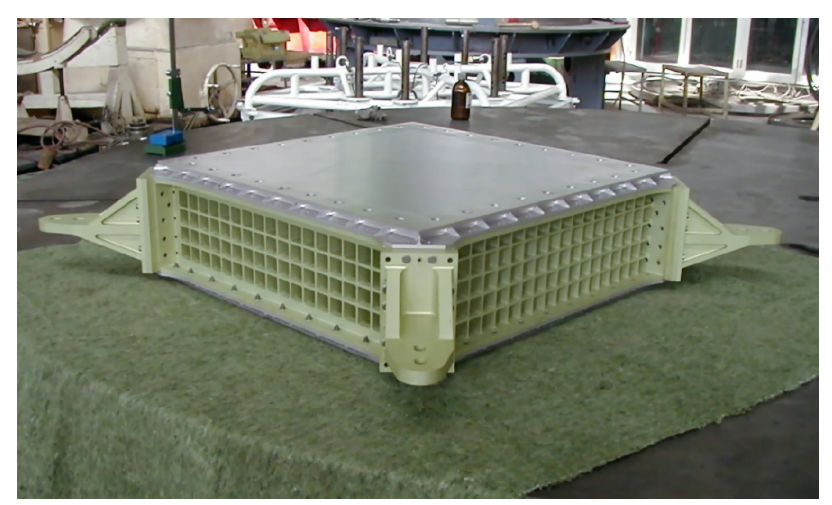

Figure 1. ECAL for the AMS-O2 experiment inside the supporting structure. 324 photomultipliers will be housed in the lateral panels. The electromagnetic shower is sampled in $\mathrm{X}$ and Y directions by scintillating fibers glued to the inside of the grooved lead foils.

The major challenge for the photomultiplier and for its front-end electronics is related to the very large dynamic range of light pulses created in optical fibers by cosmic rays. The signal in the photomultiplier ranges from a few photoelectrons for minimum ionizing particles (MIP) to about $10^{5}$ photoelectrons for electromagnetic showers corresponding to very high energy particles (for instance an electron of $1 \mathrm{TeV}$ energy $)^{5}$. Power consumption for all ECAL electronics (including PMT bleeders) is limited to $100 \mathrm{~W}$. 
Another challenge comes for mechanics of the ECAL. The total weight is limited to $630 \mathrm{~kg}$ (the weight of the Lead + the fibers is $512 \mathrm{~kg}$ ), first resonance frequency of the structure must be higher than $50 \mathrm{~Hz}$ and it has to support vibrations and accelerations up to $27 \mathrm{~g}$ during the space qualification tests. All elements have to be designed to support 30000 thermal cycles during 5 years of orbiting. The ECAL will be dipped into the stray magnetic field of the superconducting magnet, ranging up to 300 Gauss. The weight budget allowed for the detector and the space available between photomultipliers are very limited, which implies a fine optimization between the response of the photomultipliers on the magnetic field and the design of the magnetic shielding.

In the following sections we will present the performances of the photomultiplier and its front end electronics and the design of the magnetic shielding and light collection system incorporated into the mechanical structure.

\section{Photomultiplier and its front end electronics}

The total of 324 photomultipliers R7600-00-M4 from Hamamatsu will be used. The resistance for vibrations, resistance for magnetic field, square form, compactness and low weight were the major factors leading to the choice of this space qualified photomultiplier.

The properties of R7600-00-M4 were extensively studied in order to optimize its dynamic range ${ }^{4}$. As mentioned in the introduction, the expected light signal from the calorimeter ranges from a few photoelectrons to $10^{5}$ photoelectrons. Several types of bleeders were tested and the dynamic range for each bleeder were determined using the LED light pulses. The deviation from the linearity was detected by comparing the PMT response for the signal of two LEDs flashing simultaneously with the sum of responses to LEDs flashing individually. As can be seen in Figure 2, for a given bleeder and given high voltage, the saturation occurs at the same output charge for all four pixels of the PMT.

Figure 3 shows the relation between the number of photoelectrons at the saturation point and the gain of the photomultiplier for two different bleeders. One can observe that the saturation point differs by a factor of 5 between these two bleeders. Choosing the saturation at the level of $10^{5}$ photoelectrons and B type bleeders(see reference ${ }^{5}$ for detail) sets the gain at $10^{5}$.

It was checked that in these conditions the signal corresponding to about 9 photoelectrons (expected for MIPs) can be clearly separated from the background (Figure 4 ).

As a result, the design of the front end electronics (dedicated ASIC chip) was made to accept signals from $30 \mathrm{fC}$ to $2 \mathrm{nC}^{6}$. In this front end chip signals from PMT anodes are separated to low and high amplitude parts (ratio of 


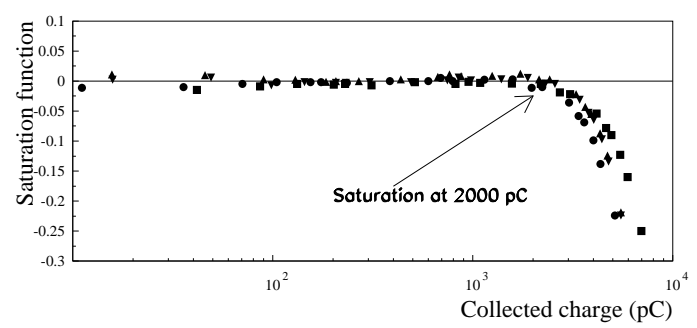

Figure 2. The saturation of the response of the photomultiplier as a function of the collected charge (B type HV bleeder and voltage supply of $-600 \mathrm{~V}$ - see text).



Figure 3. Relation between the number of photoelectrons at saturation point and the gain of the photomultiplier for two different high voltage bleeders.



Figure 4. The separation of the signal and the pedestal as a function of the applied high voltage. The natural fluctuation of the signal corresponding to 8.5 photoelectrons corresponds to $\mathrm{RMS}=2,9$.

about 30), than shaped and integrated with $2 \mu$ s time constant and finally treated by track and hold logic. The signal from last dynode is also proceed 
by low gain channel. Finally the multiplexer incorporated in the chip sends these 9 signals to the ADC. The whole system (including ADC) is placed on the small electronic board directly coupled to the bleeder board. The linearity tests performed on the first version of the chip are presented in Figure 5 . The right linearity was obtained in the whole expected range and the level of noise is compatible with the detection of MIPs.

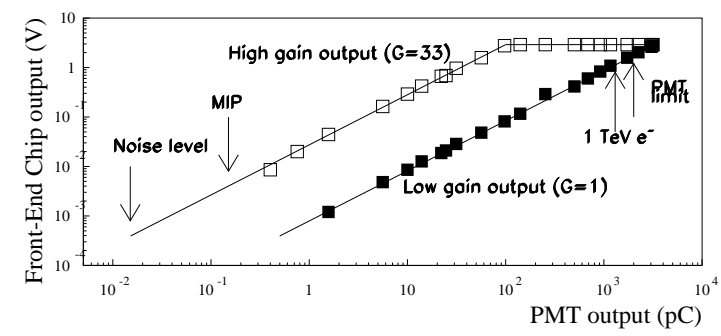

Figure 5. The output signal of the dedicated front end ASIC chip as a function of the signal delivered by the photomultiplier. One can observe a good linearity in the whole required dynamic range and the noise at the level of $10 \%$ of the MIP signal.

\section{Magnetic shielding and light collection system}

As it was mentioned in the introduction, the stray magnetic field from the AMS-02 superconducting magnet is of the order of $200-300$ Gauss in the region where the calorimeter is placed. The limit of the weight and the small space available between photomultipliers makes it necessary to optimize the thickness of the material used for magnetic shielding. Finite element calculations corresponding to this optimization can be found in reference ${ }^{7}$. In these calculations it was shown that when taking into account the global configuration of magnetic materials (those for both RICH and the calorimeter), the magnetic field can increase by a factor of 2 in some particular regions, where the density of surrounding magnetic materials is important. This is due to the attraction of magnetic field lines by the structure. Taking into account this effect, the local configuration of the magnetic shielding was calculated. It was shown that the shielding of photomultipliers by $1 \mathrm{~mm}$ thick, square soft iron tubes (section of $2.4 \mathrm{~cm}$ by $2.4 \mathrm{~cm}$ and length of $7 \mathrm{~cm}$ ) lowers the magnetic field in the tube down to 10 Gauss in the central part. The measurements performed on some particular configurations confirmed the validity of calculations. It was also checked, that the response of the photomultiplier is affected 
by less than $10 \%$ by such a field ${ }^{4}$.

Finally, the light collection system between the fibers of the calorimeter and the photomultiplier was optimized: leaving the fiber of the calorimeter, the light crosses successively a RTV joint, $30 \mathrm{~mm}$ Plexiglas conical light guide, a second RTV joint and enter the photomultiplier. The collection efficiency of this set up was measured to be about $70 \%$.

\section{Conclusions}

The optimization of the processing of the light signal from the electromagnetic calorimeter of the AMS-02 experiment is described. The extensive study of the R7600-00-M4 photomultiplier from Hamamatsu was done leading to following conclusions:

(1) the high voltage bleeder of the type B from Hamamatsu allows the collection of signals in the whole required dynamic range (from $310^{-14}$ C to

$210^{-9} \mathrm{C}$, corresponding to MIP and to electromagnetic showers of 1 $\mathrm{TeV}$ electron respectively) with the gain of $10^{5}$.

(2) the magnetic field is required to be less than 10 Gauss to limit the signal reductionto $10 \%$.

The dedicated front-end ASIC chip was designed and the linearity of the chip was tested in the whole required dynamic range. The noise level was measured at the level of $10 \%$ of the MIP signal. The magnetic shielding of the photomultipliers was optimized by finite element calculations. It was shown that $1 \mathrm{~mm}$ thick soft iron tubes lower the magnetic field from 200-300 Gauss down to about 10 Gauss for the whole geometry of the calorimeter. Finally, the light collection system between the calorimeter and the photomultiplier was designed (RTV joints and conical light guides made with Plexiglas).

\section{References}

1. http://ams.cern.ch/AMS and references quoted there.

2. B.Alpat - AMS on ISS - talk given on the Conference Frontiers Detectors for Frontier Physics - 8th Pisa Meeting on Advanced Detectors, May 2000.

3. Hamamatsu data sheet, March 1997.

4. R.Kossakowski et al, LAPP-EXP-2002-02 / AMS Note 2002-01-03.

5. Monte Carlo simulations with Geant 4 - AMS collaboration

6. V.Hermel - EMC electronics status - presentation on AMS Technical Interchange Meeting, CERN, Juin 2001.

7. F.Cadoux, R.Kossakowski, J.P.Vialle - AMS Note 2001-05-01. 